\title{
Anaortic, off-pump coronary artery bypass using multiple arterial grafts: Surgical technique
}

\author{
Michael Seco ${ }^{1,2}$, Fabio Ramponi ${ }^{1,3}$, R. John L. Brereton ${ }^{4}$, Greg A. Bigelow ${ }^{5}$, Asvin M. Ganapathi ${ }^{5}$, Michael P. \\ Vallely \\ 1Sydney Medical School, University of Sydney, Sydney 2006, Australia. \\ ${ }^{2}$ Department of Cardiothoracic Surgery, Macquarie University Hospital, Sydney 2109, Australia. \\ ${ }^{3}$ Department of Cardiothoracic Surgery, Royal Adelaide Hospital, Adelaide 5000, Australia. \\ ${ }^{4}$ Department of Cardiothoracic Surgery, Royal North Shore Hospital, Sydney 2065, Australia. \\ ${ }^{5}$ Division of Cardiac Surgery, The Ohio State University Wexner Medical Center, Columbus, OH 43210, USA.
}

Correspondence to: Dr. Michael P. Vallely, Division of Cardiac Surgery, The Ohio State University, Wexner Medical Center, N805 Doan Hall, 410 West 10th Avenue, Columbus, OH 43210, USA. E-mail: michael.vallely@osumc.edu

How to cite this article: Seco M, Ramponi F, Brereton RJL, Bigelow GA, Ganapathi AM, Vallely MP. Anaortic, off-pump coronary artery bypass using multiple arterial grafts: Surgical technique. Vessel Plus 2021;5:18. https://dx.doi.org/10.20517/25741209.2020 .100

Received: 23 Dec 2020 First Decision: 20 Jan 2021 Revised: 27 Jan 2021 Accepted: 5 Feb 2021 Available online: 17 Apr 2021

Academic Editor: Cristiano Spadaccio Copy Editor: Xi-Jun Chen Production Editor: Xi-Jun Chen

\begin{abstract}
Traditional on-pump coronary artery bypass grafting on an arrested heart using a single arterial graft carries 2 main potential drawbacks: the risk of perioperative neurological injury and the known failure rate of vein grafts. To address this, we describe a surgical technique of anaortic, off-pump coronary artery bypass that avoids all manipulation of the ascending aorta and uses multiple-arterial grafts to achieve complete revascularisation. This provides optimal short- and long-term outcomes and is particularly important in high-risk subgroups. The standard graft configuration is the left internal mammary artery to the anterior wall, and the right internal mammary artery is extended with the radial artery and brought through the transverse sinus to revascularise the lateral and inferior walls sequentially. Alternative configurations, including "T" grafts and using long saphenous vein, are considered if patients have factors limiting arterial conduit selection. Arterial conduits are harvested using a skeletonised technique. The radial artery may also be harvested endoscopically. Wide, bilateral retrothymic tunnels are formed for the internal mammary arteries. The pericardium is opened using specific incisions designed to facilitate positioning of the heart whilst maintaining venous return and cardiac output. There are 4 main positions for the heart during grafts (high-lateral wall, low-lateral wall, inferior wall and anterior wall). These are obtained using a combination of table position, wet sponges, two nylon sutures placed in the pericardium and the off-pump stabiliser. All distal anastomoses are performed using homemade intra-coronary silastic shunts, which provide optimal grafting conditions. Graft patency is confirmed using transit-flow time measurement.
\end{abstract}


Keywords: Anaortic, off-pump, coronary artery bypass, arterial grafting, radial artery, internal mammary artery

\section{INTRODUCTION}

The most widely utilised coronary artery bypass grafting (CABG) technique remains the left internal mammary artery (LIMA) to the left anterior descending (LAD) artery and reversed long saphenous vein (LSV) from the aorta to other arteries, performed using cardiopulmonary bypass on an arrested heart. However, there are 2 significant drawbacks to this widely performed technique.

Firstly, there is now an established relationship between the degree of aortic manipulation and the risk of neurological injury. In a large network meta-analysis performed comparing all techniques (on-pump CABG with an aortic cross-clamp, off-pump CABG with a partial occlusion clamp, off-pump CABG with a proximal anastomotic device and "anaortic" off-pump CABG with no aortic manipulation), the anaortic technique was shown to be the most effective method for decreasing the risk of stroke (52\%-78\% reduction compared to the other techniques ${ }^{[1]}$. It also reduced the risk of mortality, renal failure, bleeding complications and other morbidity ${ }^{[1]}$. The European myocardial revascularisation guidelines now recommend minimisation of aortic manipulation where possible for all patients (Class I, Level B), and particularly in high-risk subgroups (Class IIa, Level B) and those with significant aortic atherosclerosis (Class I, Level B) [Figure 1] ${ }^{[2]}$.

There is also growing recognition and understanding of more subtle neurocognitive injury and psychiatric issues following CABG. The aetiologies of these have been linked to silent brain infarcts, cerebral hypoperfusion, micro-emboli and a systemic inflammatory response, which are often associated with cardiopulmonary bypass and aortic manipulation ${ }^{[3]}$. There are ongoing studies examining whether this relationship is causative and if avoidance of aortic manipulation improves these outcomes as well.

The second drawback to the traditional technique is the known failure rate, both early and late, of LSV grafts $^{[4]}$. In contrast, the benefits of multi-arterial grafting (MAG), using the right internal mammary artery (RIMA) and/or radial artery, on long-term patient survival have been known for more than 2 decades ${ }^{[5]}$. More recently, several publications have demonstrated this, including the Arterial Revascularisation Trial (as-treated analysis ${ }^{[6,7]}$ and data from large observational studies ${ }^{[8-10]}$. The European myocardial revascularisation guidelines advocate a MAG strategy for all patients with a life-expectancy greater than 5 years, including the use of bilateral internal mammary arteries (BIMA) in patients at low risk of sternal complications (Class IIa, Level B), the use of a skeletonised harvest technique in those at risk (Class I, Level B) and the use of the radial artery for high-grade stenoses (Class I, Level B) [Figure 1] ${ }^{[2]}$.

There are criticisms of both off-pump CABG and MAG strategies. Long-term observational studies of offpump CABG have found poorer survival, most likely related to higher rates of incomplete revascularisation and fewer distal anastomoses ${ }^{[1]}$. The use of BIMAs has led to higher risk of sternal wound infections when a non-skeletonised harvest technique is used ${ }^{[12]}$, and MAG strategies are generally criticised as complex and technically difficult ${ }^{[13]}$.

To address these concerns, we describe reproducible techniques to accomplish complete revascularisation, using MAG where possible, whilst completely avoiding aortic manipulation. This is achieved primarily through the use of composite grafts based off internal mammary artery inflow(s). The grafting strategy can still be customised to individual patient risk profile and considerations. 


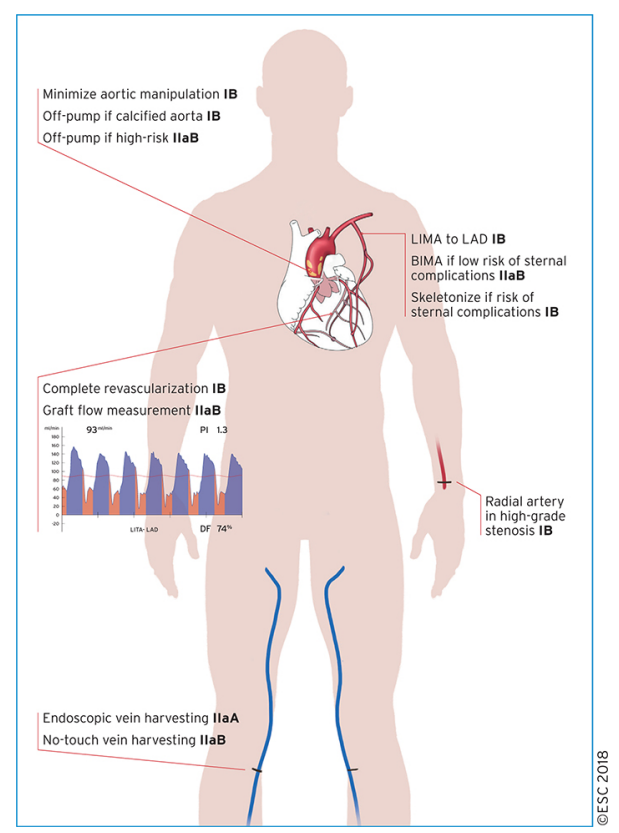

Figure 1. Specific surgical techniques recommended in the 2018 ESC/EACTS myocardial revascularisation guidelines ${ }^{[2]}$. Reprinted with permission.

\section{SURGICAL TECHNIQUE}

\section{Preoperative assessment}

All patients undergo an extensive workup preoperatively. In addition to standard investigations, patients over 70 years or those with risk factors undergo a non-contrast computed tomography (CT) scan of the chest to assess for aortic atherosclerosis ${ }^{[2]}$. All patients also undergo bilateral duplex ultrasound scans of the carotid and vertebral arteries to assess for concomitant disease. The proximal internal mammary arteries can be included in this scan and give the surgeon useful information on vessel size and potential inflow limitations. If significant carotid or vertebral disease is detected, then a CT aortogram with 4 vessel run-off is performed to assess the arch vessels and Circle of Willis in further detail. Intervention, including synchronous carotid enterectomy and CABG, is based on current guideline recommendations ${ }^{[2]}$.

An Allen's test is performed, with $<5 \mathrm{~s}$ deemed suitable to harvest the radial artery. In borderline cases, the Allen's test is performed using a pulse oximeter, and, if a pulsatile waveform is obtained and oxygen saturation in the hand remains stable, the radial artery is harvested. If not, an alternative conduit is considered.

\section{Anaesthetic considerations}

On arrival in the operating room, active warming using a forced-air warming blanket is commenced, as maintaining normothermia throughout the operation is important and patients easily become hypothermic. The patient is positioned supine with the left arm gently abducted on an arm-board.

A Swan-Ganz pulmonary artery catheter and transoesophageal echocardiography (TOE) probe is inserted in all patients. These provide useful information for maintaining cardiac output and detecting ischaemia during positioning of the heart for grafting. Hypovolaemia is poorly tolerated during this operation, and this can be detected using a combination of the TOE to observe cardiac filling, arterial systolic pressure variation and dynamic responses in central venous pressure and pulmonary artery diastolic pressure to fluid 
challenges.

A low dose milrinone infusion (0.1-0.2 $\mathrm{g} / \mathrm{kg} / \mathrm{min}$, no loading dose) is commenced at the beginning of the operation. This provides 2 key advantages: increased cardiac output to maintain end-organ perfusion during heart positioning and flow through the grafts once performed, and its systematic vasodilatory effects help prevent arterial graft spasm. For these reasons, this infusion is usually continued overnight and ceased early on Postoperative Day 1. A low dose background infusion of noradrenaline may also be required.

During the operation, boluses of intravenous beta-blockers (metoprolol 2.5-5 $\mathrm{mg}$ ) may be required to control heart rate within a range of $60-70 \mathrm{bpm}$, which is optimal for grafting.

\section{Conduit and graft configurations}

Our aim is to utilise all-arterial grafts in all patients. Our standard graft configuration for triple vessel coronary disease is an in situ skeletonised LIMA-LAD, as well as an in situ skeletonised RIMA extended with the radial artery and brought through the transverse sinus to revascularise the lateral and inferior wall vessels [Figure 2A]. This allows for all coronary targets to be adequately reached and complete revascularisation performed. It also separates the blood supply to the anterior wall from the rest of the heart, thus reducing the risk of compromising the LIMA to LAD graft. Performing multiple sequential anastomoses using the radial artery is also technically easier than using a RIMA. Passing the composite graft through the transverse sinus avoids having the graft cross the midline anteriorly, making redo sternal entry and management of mediastinitis safer.

In patients with triple vessel disease and high-grade lesions only, alternatives for complete revascularisation include in situ LIMA-LAD and LIMA-Radial or LIMA-RIMA "T" graft to lateral and inferior walls [Figure $2 \mathrm{~B}$ ]. The advantage of the LIMA-Radial composite graft is the ability to harvest both conduits simultaneously and reduce operating time, whilst the advantage of the LIMA-RIMA composite graft is the avoidance of a second incision and better sized-matched conduits.

In patients with left-sided coronary lesions only, the aforementioned "T" grafts may be used, or a in situ RIMA-LAD and in situ LIMA-lateral wall configuration is possible [Figure $2 \mathrm{C}$ ].

In patients where a diagonal vessel requires grafting, even though the vessel angle is not conducive to a sequential anastomosis using the LIMA, a short segment of radial artery can be used as a "Y" graft. This can be performed in combination with an in situ RIMA (with or without extension with the remaining radial artery) [Figure 2D].

There are circumstances where it is not possible to use additional arterial conduits or it carries substantial risk to do so. Radial arteries may not be suitable due to dystrophic calcification or inadequate ulnar artery collaterisation; the radial artery in the non-dominant arm may have been used for access for the coronary angiogram; occasionally a patient's occupation may preclude them (e.g., guitar virtuoso); or a coronary vessel with an intermediate-grade lesion requires grafting. Use of the BIMAs may also be considered unacceptably high risk in obese and poorly controlled diabetic patients.

In these patients where both the radial artery and RIMA are not suitable for use, there are 2 options for maintaining an anaortic technique. First, a short segment only $(3-4 \mathrm{~cm})$ of in situ RIMA may be harvested and the vessel extended with a longer segment of LSV [Figure 3]. This preserves the majority of the RIMA blood flow to the sternum via intercostal vessels but provides some of the theoretical advantages of a second 

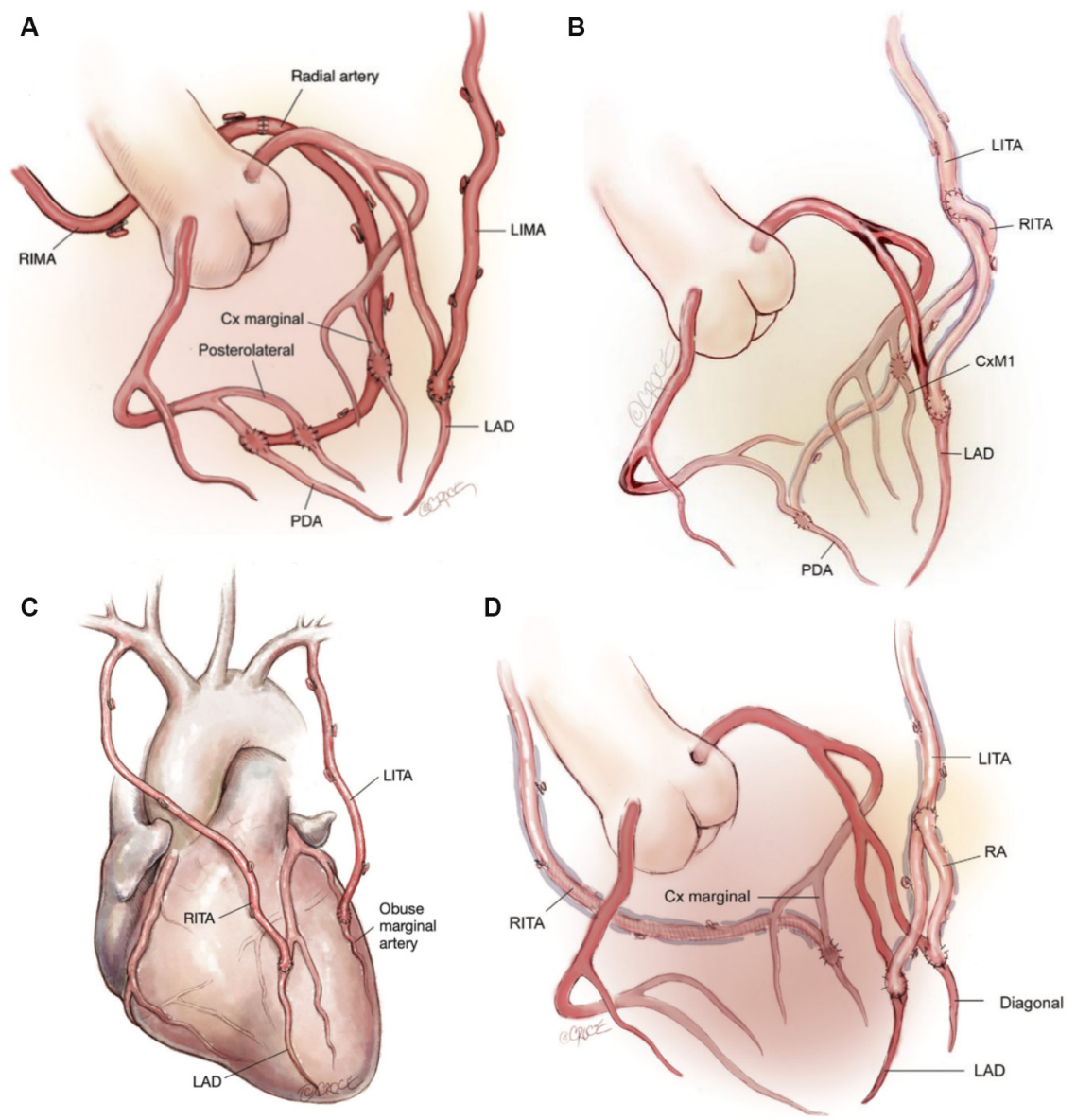

Figure 2. Multiple-arterial grafting strategies that maintain an anaortic technique: (A) in situ LIMA-LAD and in situ RIMA-lateral-inferior walls ${ }^{[14]}$; (B) in situ LIMA-LAD and LIMA-RIMA or LIMA-Radial T Graft-lateral-inferior walls ${ }^{[15]}$; (C) in situ RIMA-LAD and in situ LIMAlateral wall ${ }^{[16]}$; and (D) in situ LIMA-LAD, LIMA-Radial Y graft-diagonal and RIMA-lateral wall ${ }^{[15]}$. Figures reprinted according to CC BYNC-ND 4.0 license. RIMA: Right internal mammary artery; LIMA: left internal mammary artery; LAD: left anterior descending; PDA: posterior descending artery; LITA: left internal descending artery; RITA: right internal descending artery; RA: right atrium.

in situ graft and avoids the need for a proximal aorto-venous anastomosis. Alternatively, a LIMA-LSV "T" graft can also be used.

\section{Conduit harvesting}

\section{Internal mammary artery harvesting}

The internal mammary arteries are harvested using a skeletonised technique [Figure 4]. This reduces the risk of deep sternal wound infection during BIMA harvest ${ }^{[12]}$, minimises chest wall trauma and achieves maximum conduit length. A Rultract Skyhook (Rultract, Cleveland, USA) is used to retract the sternum as this provides optimal exposure. The internal mammary pedicle is exposed using a combination of diathermy and blunt dissection using forceps and a Ray-Tec to "push" the pleura laterally. Keeping the pleural curtain intact has benefits during the operation in that the lungs are kept out of the field. The vessel is also kept extra-pleural, which prevents avulsion injuries when the lungs are inflated and keeps it away from the lung in the event that the patient needs a lobectomy in the future.

The endo-thoracic fascia is carefully incised directly below and along the length of the artery. A combination of low power diathermy $(\leq 20 \mathrm{~W})$, Pott's scissors and gentle traction on the fascia using long 

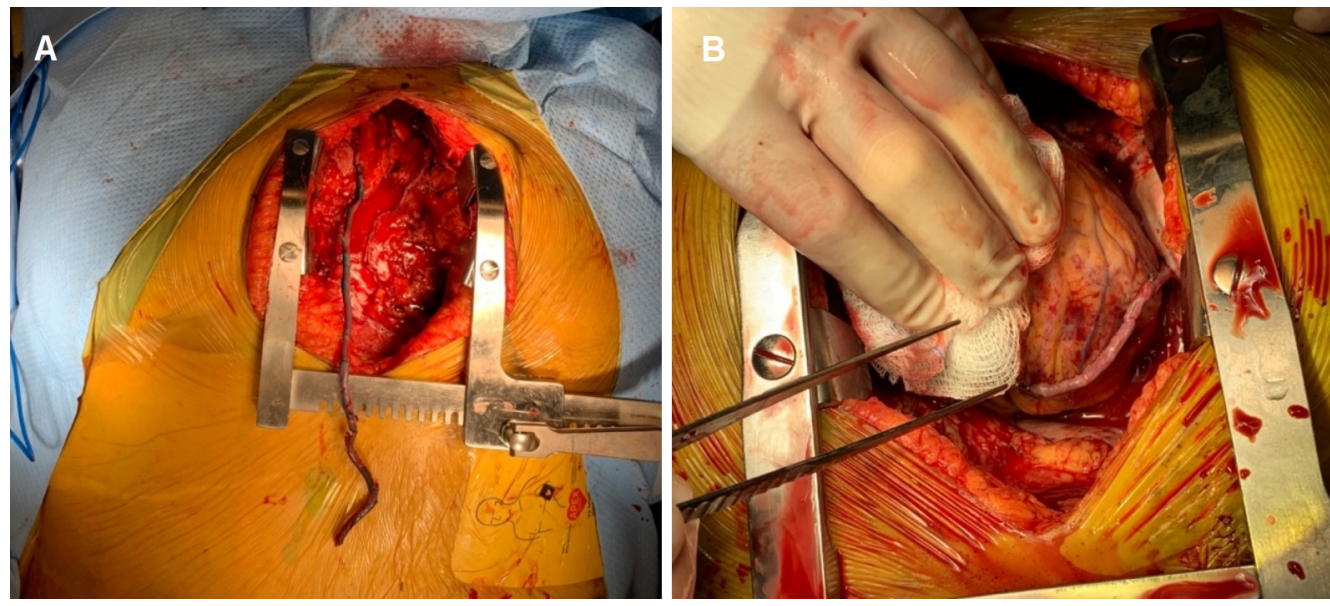

Figure 3. Short segment of in situ right internal mammary artery is extended with long saphenous vein (A) and used to graft lateral and inferior walls of the heart (B).

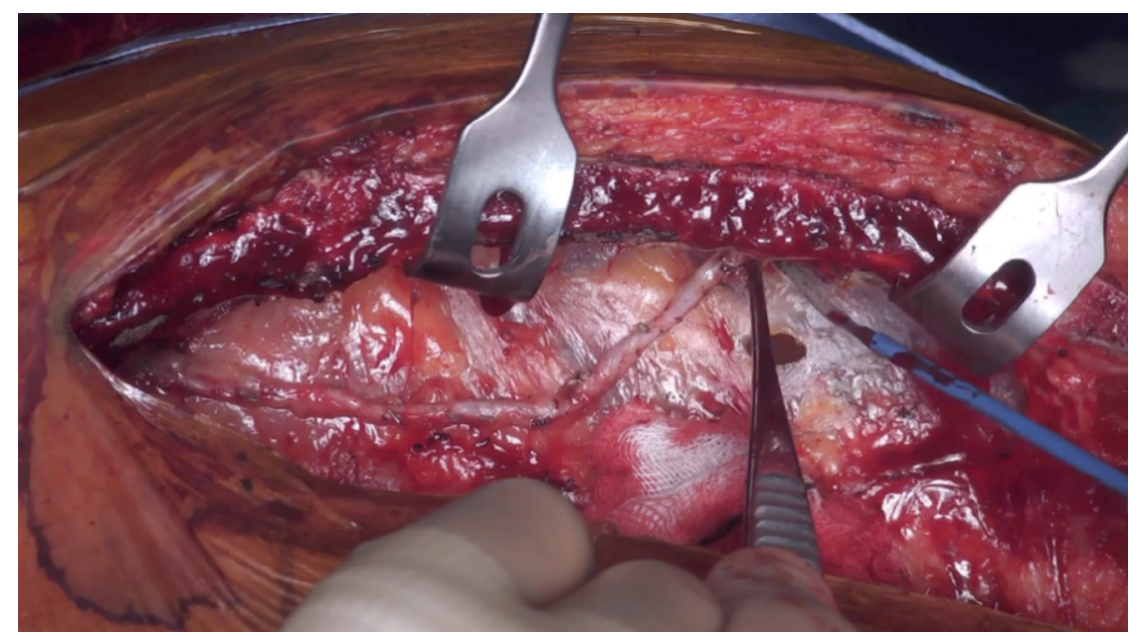

Figure 4. Left internal mammary artery harvest using a fully skeletonised technique. The harvest is performed extra-pleurally where possible.

Dietrich's forceps is used to expose the vessel fully. The medial internal mammary vein at level of the subclavian vein is divided, as this improves the final length and lie of the conduit and makes proximal harvest safer. Care must be taken not to injure the phrenic nerves at this level, especially when harvesting bilateral internal mammary arteries.

The artery is then harvested from above the left subclavian vein, down to its bifurcation. The branches are divided using clips and/or low power diathermy. Alternatively, the Harmonic Scalpel (Ethicon, Ohio, USA) may be used. Both left and right internal mammary arteries are harvested using this technique. After systemic heparinisation, the vessels are divided distally, sprayed with papaverine solution and wrapped in papaverine soaked gauze until ready for grafting.

If a reversed LSV graft is being used to extend an in situ RIMA as a composite graft, then only a short segment of RIMA needs to be harvested. The LSV conduits are usually significantly longer than the radial, and the size of the proximal RIMA is a better match to the diameter of the vein. 


\section{Radial artery harvesting}

The radial artery is harvested from the non-dominant arm or from the arm that has not been used for the coronary angiogram. An endoscopic or open harvest technique can be used. The radial artery is examined with ultrasound in the operating room prior to harvest to ensure that there is no dystrophic calcium and that the size is appropriate. If there is a problem with the radial artery, then an alternate grafting strategy is used as previously described.

An advantage of an open radial artery harvest is that a skeletonised technique can be used, which increases conduit length, allows all soft-tissue impediments to be removed to maximise conduit dilatation and improves handling when performing graft-to-graft and distal anastomoses. An incision is made on the volar aspect of the forearm along the groove of brachioradialis, from $1 \mathrm{~cm}$ proximal to the wrist crease to $1 \mathrm{~cm}$ distal to the elbow crease. This incision provides excellent exposure of the artery whilst reducing the risk of injury to the medial and lateral antebrachial cutaneous nerves.

The artery is exposed between brachioradialis and flexor carpi radialis using low power diathermy. The fascia directly overlying the artery is divided along its length using Pott's scissors, so that the 2 venae comitantes separate from the vessel. The artery is then suspended using a soft silastic loop, which provides upwards tension with minimal trauma to expose the vessel's branches [Figure 5A]. These are generally divided using low-power diathermy as far from the vessel as possible, so that they can be clipped later after fashioning the composite graft and obtaining maximum pharmacological and hydrostatic dilatation of the conduit. Only rapidly bleeding branches are controlled with a small haemoclip during the harvest. The artery is harvested between the recurrent radial and superficial palmar branches. It is then divided proximally and distally, and a slit is made in the proximal end with Pott's scissors for orientation and to prepare for the composite graft anastomosis. A $2.0-\mathrm{mm}$ soft cannula is used to slowly flush $20 \mathrm{~mL}$ of heparinised blood containing $5 \mathrm{mg} / 50 \mathrm{~mL}$ of Verapamil, and then the conduit is left to soak in the same solution. The wound is closed over a small suction drain, dressed and the arm placed back against the body.

An endoscopic radial artery harvest technique can also be used. The advantages of this technique are cosmetic and reduction of morbidity. The distal scar is less then $3 \mathrm{~cm}$ and is usually hidden beneath a watchstrap which is more palatable to patients than a full forearm scar.

Using a proximal orthopaedic tourniquet, a $3-\mathrm{cm}$ incision is made over the distal radial artery. The Vasoview system (Getinge, Gothenburg, Sweden) is used to expose the vessel along its length [Figure 5B]. The Ligasure Maryland forceps (Medtronic, Minneapolis, USA) are used to create a fasciotomy. The vessel, with the venae comitantes, is mobilised along its length and the branches divided using the Ligasure. The harvested vessel is delivered through a proximal stab incision. The vessel is then managed as previously described for the open technique.

\section{Preparation of the pericardium}

Bilateral, extra-pleural retro-thymic tunnels are developed first. This is done before the pericardium is opened as dissection is easier with this still intact. The left-sided tunnel is prepared by mobilising the thymic remnant and fat-pad off the aortic arch. Using a finger, the superior entry to the tunnel is formed below the innominate vein. There is a defined "soft-spot" above the thymus and below the innominate vein that is easily developed with the left index finger. This tunnel needs to be wide to prevent kinking of the LIMA and to ensure that the lie is optimal. It is also important to take care that there is no bleeding within the tunnel before passing the conduit through the tunnel. 

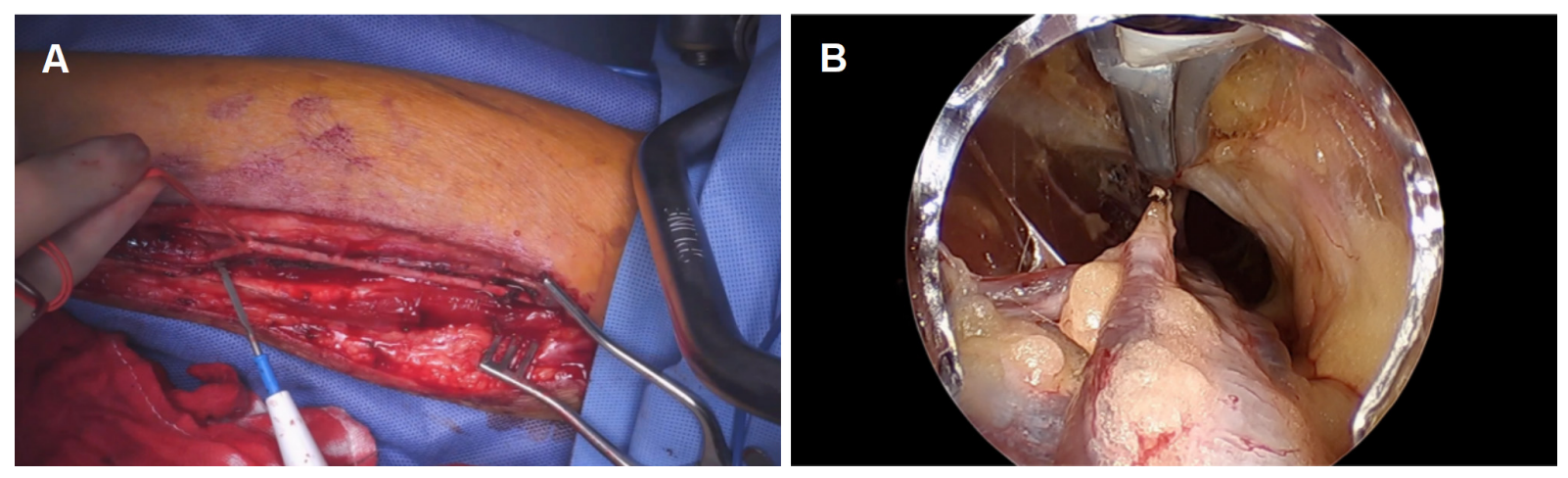

Figure 5. Radial artery harvest using: (A) an open, fully skeletonised technique; and (B) an endoscopic technique.

A right-sided tunnel is made by dissecting the thymic fat pad off the pericardium down to the superior vena cava (SVC). This tunnel needs to be mobilised superiorly and medial to the lung in an extra-pleural fashion. There are often bands of connective tissue and/or veins that need to be divided to allow an unimpeded path for the graft into the tunnel. This is important as failure to divide these adhesions can result in graft kinking, impeded flow and graft failure. The RIMA is only brought through the tunnel after the end-to-end extension with the radial artery or LSV is performed (described below).

The pericardium is opened longitudinally. It is then extended: (1) obliquely over the pulmonary root [Figure 6A]; (2) laterally and left wards at the diaphragm [Figure 6B]; (3) inferiorly down to the inferior vena cava (IVC) [Figure 6C]; and (4) at the right atrium/SVC junction [Figure 6D]. The left superior pericardial slit over the pulmonary root is important to allow the LIMA to enter the pericardium with an optimal lie. The left pericardial flap is hitched to the sternum using the retractor to improve exposure. The right-sided pericardial slits are important as they allow the heart to roll on the caval access into the right chest without significantly impeding venous return, which is needed for exposure during lateral wall grafting. Care must be taken not to injure the phrenic nerves when performing these incisions in the pericardium.

\section{Preparation of the composite grafts}

Construction of the composite grafts are performed on a cradle of moist sponges, suspended over the sternal retractor with arterial clips. Arterial anastomoses are performed using polypropylene "flat-pack" 60 $\mathrm{cm} \mathrm{7/0} \mathrm{or} \mathrm{8/0} \mathrm{sutures,} \mathrm{depending} \mathrm{on} \mathrm{the} \mathrm{calibre} \mathrm{and} \mathrm{quality} \mathrm{of} \mathrm{the} \mathrm{vessels.}$

\section{Right internal mammary artery-radial or right internal mammary artery-long saphenous vein extension graft}

The RIMA is attached to a folded wet sponge placed at the cranial aspect of the sternal wound [Figure 7]. The RIMA and radial artery or reversed LSV are bevelled to compensate for any size mismatch between the conduits. The end-to-end anastomosis is then performed. Before tightening and tying the suture, the bulldog clamp on the RIMA is moved distal to the anastomosis, which maximises tension to balance the risk of leaks and purse-stringing effect. If there is any residual vasospasm the composite graft is flushed with heparinised/verapamil blood and the outside is sprayed with papaverine solution. Any soft tissue bands across the arterial grafts are released to ensure maximum dilation of the vessels. The remaining radial artery side branches are clipped with small haemoclips.

\section{Left internal mammary artery-radial or left internal mammary artery-right internal mammary artery "T" graft}


The LIMA is pulled out to length, with a small amount of tension, within the retro-thymic tunnel and clipped with a mosquito clamp to a wet-sponge positioned on top of the aforementioned cradle. The radial artery or free RIMA is then anastomosed to the LIMA at the position of the pulmonary valve. It is important to ensure that the LIMA has no redundancy in the retro-thymic tunnel. Kinking of the LIMA can compromise the blood supply to the whole heart, which can be catastrophic.

The LIMA arteriotomy is performed on the superior surface at the position of the pulmonary valve. The lie of both ends of the composite graft depends on the correct orientation and positioning of the anastomosis. The radial artery is bevelled to facilitate an appropriate size-match to the LIMA. Care is taken to match the vessels accurately to avoid compromising the inflow and/or outflow of the grafts. The radial artery sidebranches are managed as described above.

\section{Cardiac positioning}

Two 1 Nylon sutures are placed in the pericardium to assist with cardiac positioning: (A) in the left, lateroinferior pericardial recess; and (B) in the diaphragmatic surface, just medial to the inferior vena cava [Figure 8]. Care must be taken not to injure structures deep to the pericardium during this. The left atrial appendage is inspected and can be amputated at this point. This makes it easier to pass a composite RIMAradial/LSV graft via the transverse sinus and to perform anastomoses on the intermediate and high obtuse marginal arteries. This may also reduce the risk of perioperative stroke in patients with atrial fibrillation, although evidence is limited ${ }^{[17]}$.

The RIMA-radial/LSV composite graft is then brought through the right retrothymic tunnel and over the SVC into the pericardium. It is then passed through the transverse sinus to the left side - a Semb or Satinsky clamp is used to grasp the tip of the graft - and then the heart is placed in its natural position, before gently pulling the graft through. This is to reduce tension on the graft and prevent dislodgement of haemoclips. Correct orientation of the graft is then checked with visual inspection. It is important to inspect the graft-tograft anastomosis at this point to check that there is no bleeding.

Next, the heart is positioned for grafting. There are 4 major positions used [Figure 9A-D].

(1) High-lateral wall vessels (Diagonal 1, Intermediate, Obtuse Marginal 1 arteries). The Trendelenburg position is used, and the table is rolled towards the surgeon. A wet sponge is placed behind the heart into the oblique sinus. Tension is placed on pericardial suture A [Figure 8] to rotate the lateral wall anteriorly and towards the right chest. The stabiliser is attached to the right side of the retractor.

(2) Low-lateral wall vessels (Obtuse Marginal 2/3, Posterolateral Circumflex arteries). The table and wet sponge remain in the same position as for high-lateral wall vessels. Tension is placed on pericardial suture A across the arm of the sternal retractor and on pericardial suture B [Figure 8] inferiorly. This keeps the heart rolled towards the right chest while raising it cranially to improve the view of the lower targets. The stabiliser remains attached to the right side of the retractor.

(3) Inferior wall vessels (Posterolateral Right and Right Posterior Descending arteries). The table is centred, remaining head-down. A wet sponge is placed medial to the IVC. Tension is placed on pericardial suture B. This positions the heart "apex-up". If the heart moves to the left, then lateral tension on pericardial suture B and an additional wet sponge in the left lateral pericardial recess can help correct this. The stabiliser is attached to the arm of the retractor. 

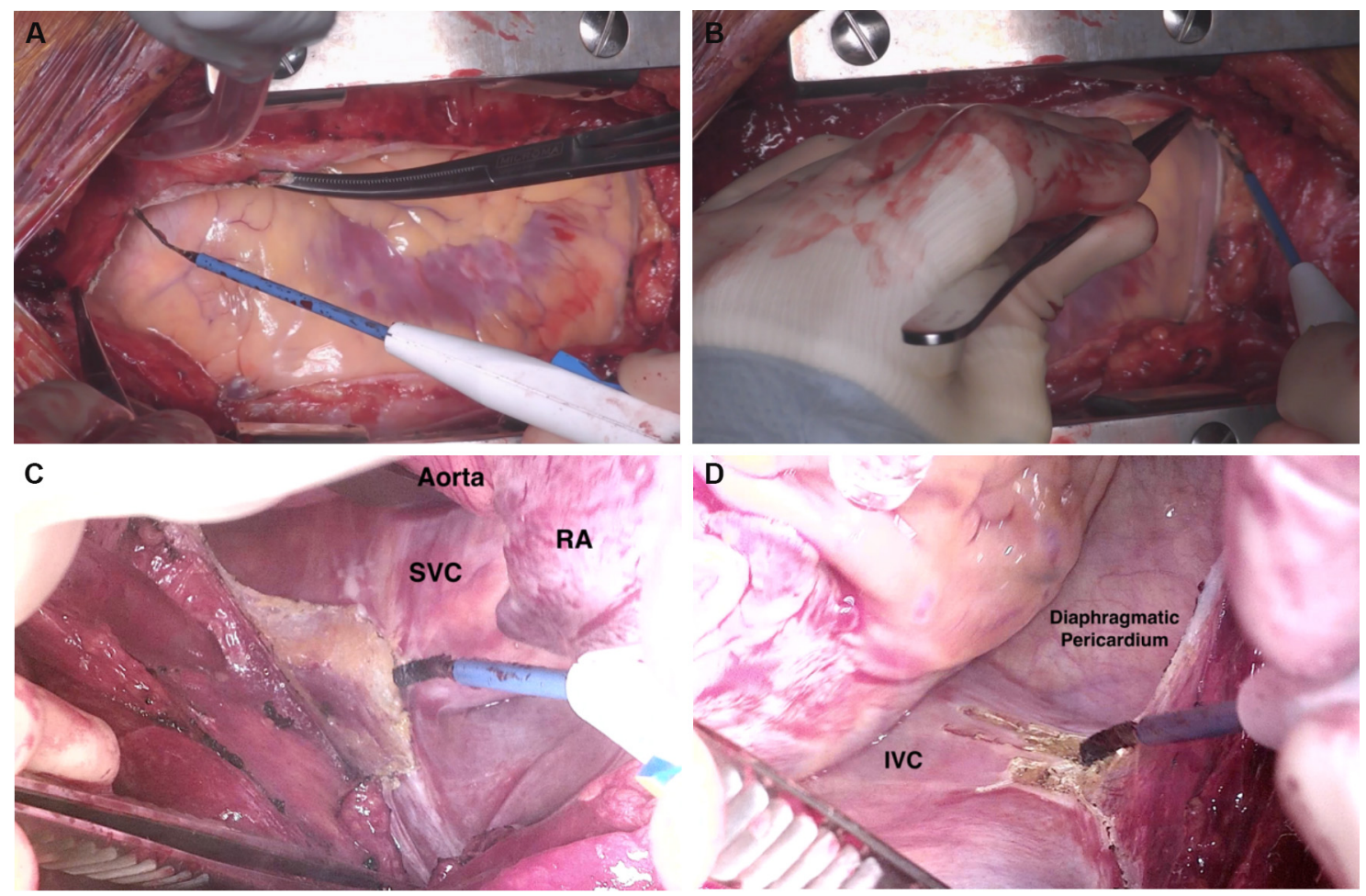

Figure 6. Preparation of the pericardium: (A) incision in left superior pericardium overlying pulmonary valve and extending obliquely; (B) incision in left inferior pericardium extended laterally; (C) incision in right superior pericardium extending downwards towards the SVC junction; and (D) incision in right inferior pericardium extending downwards towards IVC. SVC: Superior vena cava; RA: right atrium; IVC: inferior vena cava.

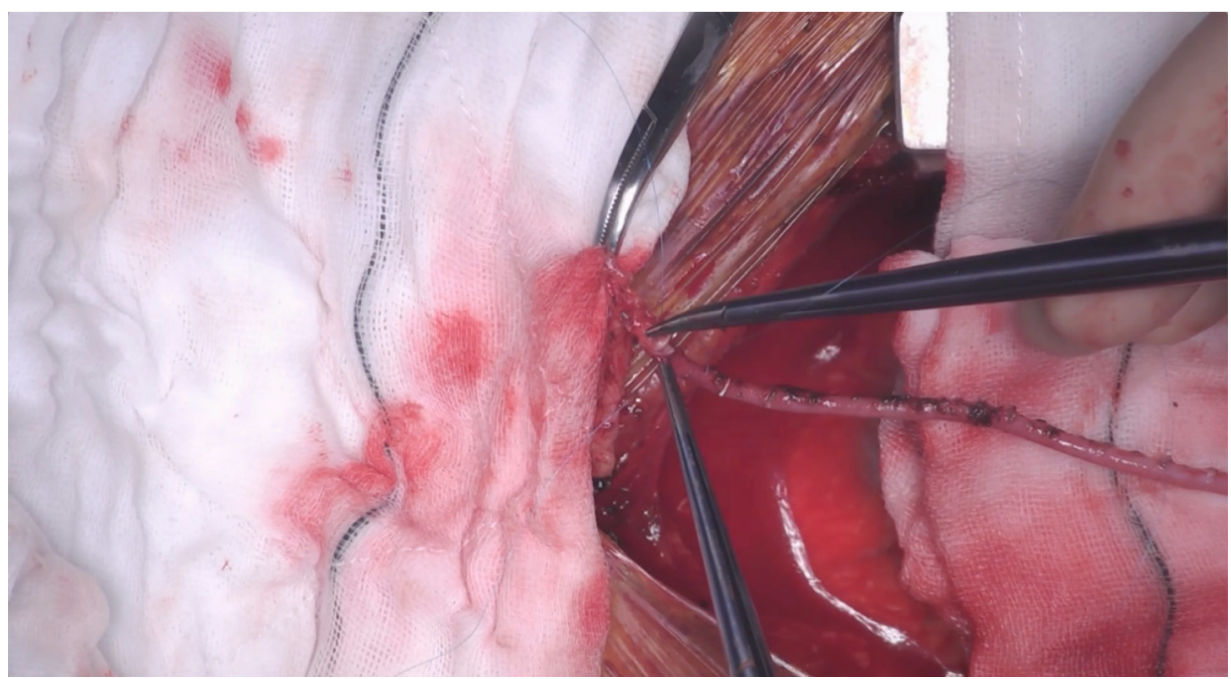

Figure 7. Right internal mammary artery-radial artery end-to-end extension anastomosis. The radial artery is placed on a cradle formed by a wet sponge over the sternal retractor.

(4) Anterior wall vessels (Diagonal 2/3, Left Anterior Descending). The table is set to the neutral position. A wet sponge is placed behind the heart, and often this is all that is required. Some lateral tension on pericardial suture A may also be helpful. The stabiliser remains attached to the arm of the retractor. 


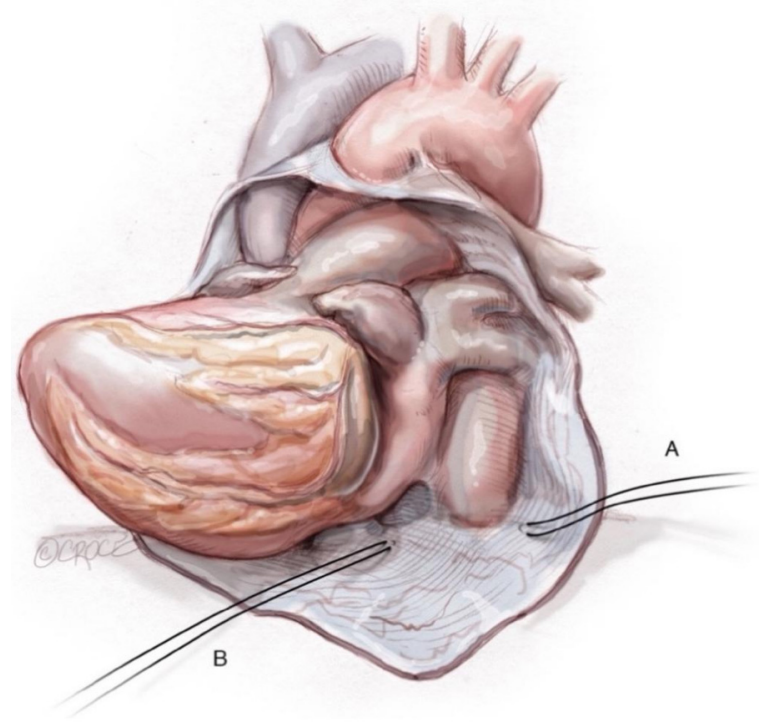

Figure 8. Pericardial sutures are placed in: (A) the left, latero-inferior recess; and (B) the diaphragmatic surface, medial to the inferior vena cava ${ }^{[14]}$. Figures reprinted according to CC BY-NC-ND 4.0 license.
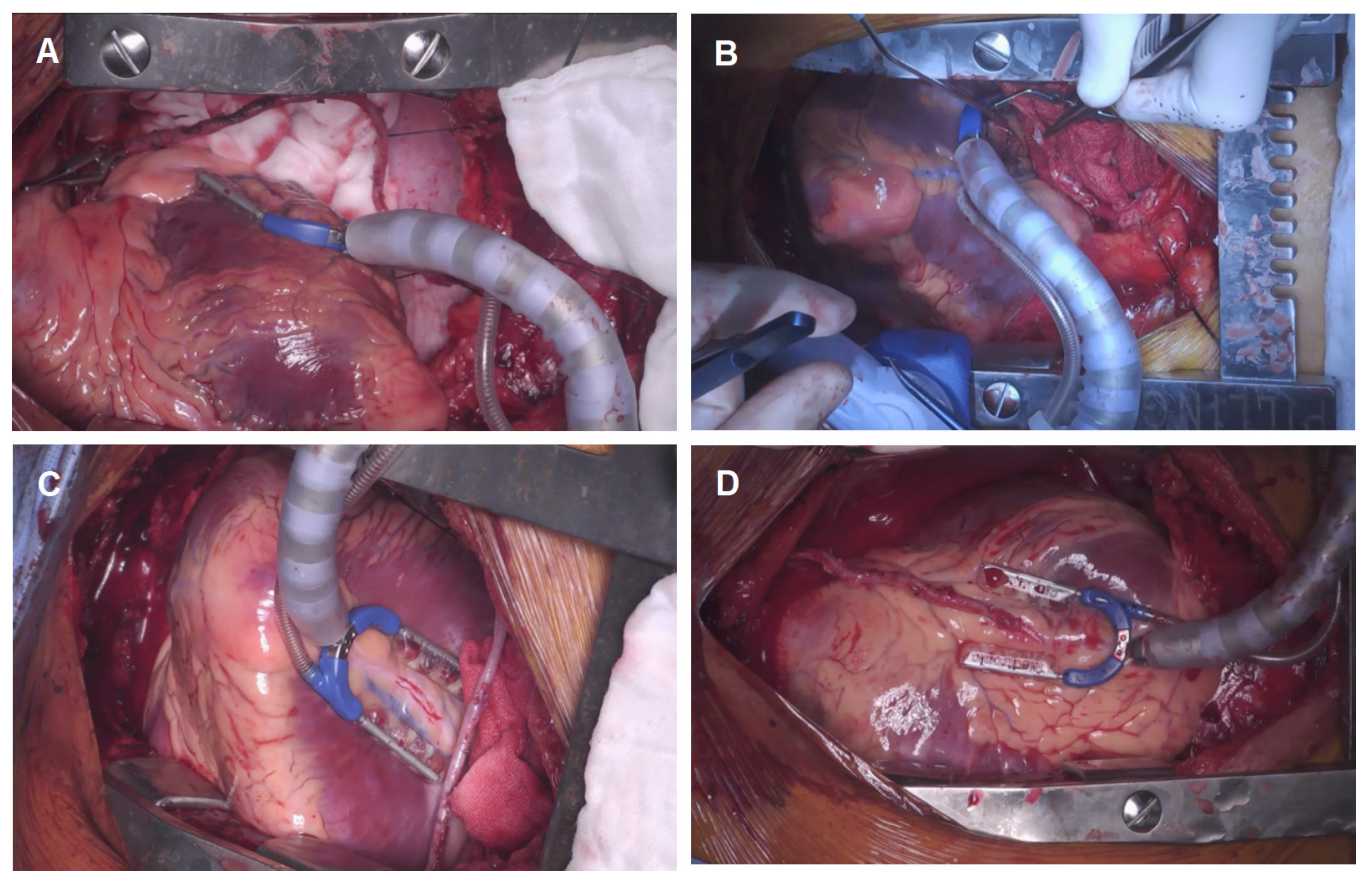

Figure 9. The 4 major cardiac positions for performing off-pump anastomoses: (A) high lateral wall vessels; (B) low lateral wall vessels; (C) inferior wall vessels; and (D) anterior wall vessels.

To provide optimal lie for RIMA-radial/LSV composite grafts, it is important to assess where each anastomosis will be before commencing. Marking the graft and coronary artery with a marking pen can be helpful. It is not necessary to leave excess length on the graft, as it will loosen when the sternum is closed. In fact, excessive length can result in kinking between the sequential anastomoses. 


\section{Grafting}

The anastomoses are usually performed in the order of the 4 positions listed above (high-lateral wall first and anterior wall last). In some cases, it may be necessary to graft the anterior wall first to improve contractility in this territory and maintain cardiac output during positioning for the remaining grafts. The anastomoses are performed using an Octopus Evolution (Medtronic, Minneapolis, USA) stabiliser and silastic intra-coronary shunts [Figure 10] ${ }^{[18]}$. The latter are "homemade" by trimming a section of silastic tubing and passing a 5/0 silk suture through it. Generally, 2 diameters of tubing are available (for large and small coronary vessels), and 3 lengths (short, medium and long) of each are prepared at the beginning of the case. The optimal shunt can then be selected for the target. Shunts provide a number of important advantages: they create a bloodless field for the anastomosis; help to prevent technical errors such as catching the back wall of the coronary artery with a suture; and provide distal coronary blood flow during grafting to maintain contractility of that territory ${ }^{[18]}$. We prefer these shunts over commercially available shunts because their length can be adjusted, and a 7/0 polypropylene suture can be pulled through the soft silastic tubing if it is accidently caught during grafting. We have not experienced intimal damage from these soft, silastic shunts before. Other aids to grafting include silastic slings or bulldog clamps placed proximally on the coronary vessel; however, these are used sparingly as there is potential to damage the vessel. If a sling is used, then a shunt is placed first, and the sling is tightened gently around the shunt to reduce endothelial injury and maintain some distal perfusion.

When using a RIMA-radial composite graft, longitudinal sequential side-to-side anastomoses are performed on the high- and low-lateral walls, and perpendicular sequential side-to-side anastomoses on the inferior wall. After the last anastomosis on the inferior wall is complete, the end of the graft is double-clipped and secured to the epicardium with a 7/0 polypropylene suture. When using a LIMA-RIMA/radial "T" graft, the orientation of the graft results in perpendicular sequential side-to-side grafts being used on the high- and low-lateral walls instead. On the anterior wall, an end-to-side anastomosis is used for the LAD artery.

All grafts are checked with transit-time flow measurement (TTFM) using the Medistim device (Medistim, Oslo, Norway). This is performed after each anastomosis is completed, again after all anastomoses are completed and finally after the heparin has been reversed. Acceptable parameters for a left-sided graft are a mean graft flow of $\geq 15 \mathrm{~mL} / \mathrm{min}$ (though preferably $\geq 30 \mathrm{~mL} / \mathrm{min}$ ), pulsatility index of $\leq 3.0$ and diastolic flow $>50 \%{ }^{[19]}$; however, these values need to be taken in the context of target vessel location, size, stenosis severity and graft diameter. Assessment of intraoperative graft patency is imperative in off-pump surgery, as there is greater potential for anastomotic issues, and these will negatively affect the patient's long-term outcome. Routine graft flow measurement is also endorsed in the 2018 ESC/EACTS myocardial revascularisation guidelines (Class IIa, Level B) ${ }^{[2]}$. Further discussion of TTFM during off-pump surgery is available $e^{[19,20]}$.

After grafting is complete, the heparin is reversed. The pericardium is partially apposed, ensuring all grafts are covered by tissue in the event of mediastinitis or if repeat sternotomy is required in the future. The total graft flows are again checked by applying the Medistim probe on the internal mammary arteries above the thymus. This also confirms that pericardial apposition has not compromised flow. Epicardial pacing wires are used only if there were rhythm disturbances during the operation. Drains are placed in the mediastinum and both pleural spaces. The sternum is closed in routine fashion. The milrinone infusion is continued overnight until the morning of Postoperative Day 1 as described. All patients receive aspirin on Postoperative Day 1 and clopidogrel once postoperative bleeding has settled $(<100 \mathrm{~mL} / \mathrm{h})$. Clopidogrel is continued for 3 months postoperatively as patients remain hypercoagulable for an extended period of time, particularly if they have had a preoperative myocardial infarction ${ }^{[21]}$. Calcium channel antagonists may also be used in patients who have received a radial artery graft ${ }^{[22]}$. 

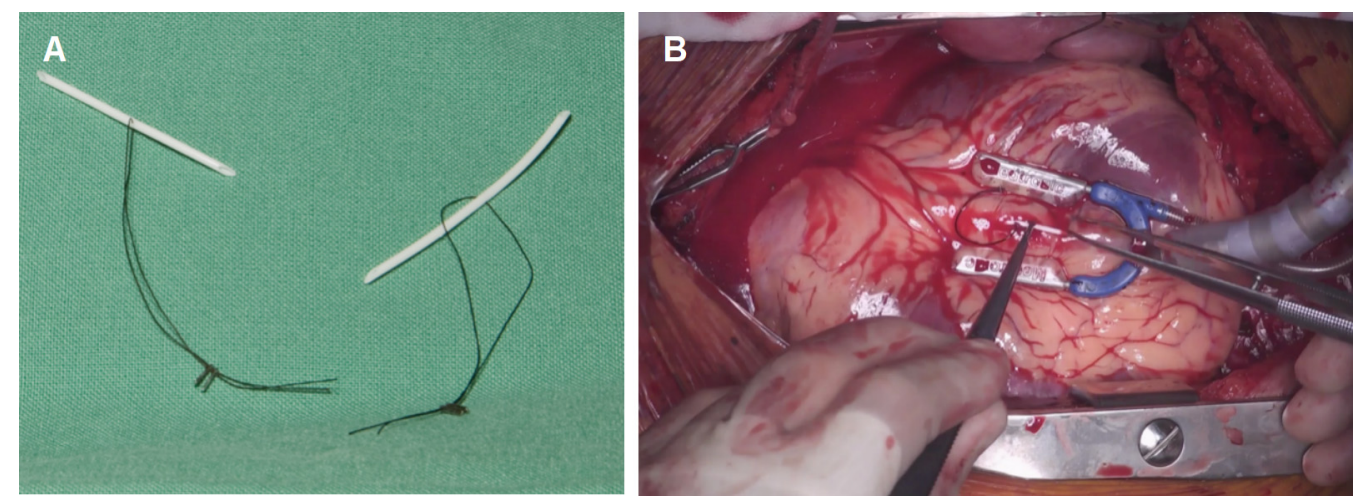

Figure 10. Intra-coronary silastic shunts: (A) preparation; and (B) insertion. They are prepared at the beginning of each case by trimming a section of silastic tubing and passing a silk stitch through it. They have an advantage over commercial shunts by being relatively inexpensive; the length can be adjusted as desired; and the silastic tubing is soft and flexible, aiding insertion.

\section{Beating-heart on-pump}

The specific pericardial releases and cardiac positioning used aim to prevent haemodynamic instability during grafting. However, in some patients this may still occur, and it is a common cause of conversion from an off-pump technique. This is significantly more likely in patients with recent ischaemic instability, severe left main coronary artery disease, large hearts and impaired preoperative ventricular function, as well as in morbidly obese patients with hypertension and diastolic dysfunction. As mentioned above, grafting the anterior wall first may be helpful. However, some patients may still require cardiopulmonary support. It is ideal to have a primed cardiopulmonary bypass machine in the operating room in these cases. In this scenario, it is still possible to avoid cross-clamping the aorta to minimise manipulation. The ascending aorta is assessed with TOE and epiaortic ultrasound for a suitable cannulation site. If there is significant atheroma, then the right axillary artery is used instead. The heart is left beating, and the conduit configuration and anastomoses are performed in the same manner as described. If the left ventricle distends during positioning, then a vent is placed in the right superior pulmonary vein.

\section{CONCLUSION}

In an era of increasingly high-risk patients and advanced percutaneous intervention options, a standardised CABG technique that reduces neurological injury and other short-term complications whilst also improving long-term survival is needed. In this article, we describe reproducible techniques that avoid all manipulation of the ascending aorta and use multiple arterial grafts to achieve complete revascularisation. This technique can be adjusted for individual patient factors.

There has been a quiet ground swell of opinion that coronary artery deserves the same level of attention as the other sub-specialty areas of cardiac surgery, including mitral and aortic surgery ${ }^{[23]}$. Anaortic, off-pump, multi-arterial CABG is technically more demanding than the traditional technique, and thus requires dedicated training and a stepwise introduction.

\section{DECLARATION}

\section{Authors' contributions}

Development of surgical techniques described: Vallely MP, Brereton RJL

Significant contribution to writing of manuscript: Seco M, Ramponi F, Vallely MP 
Revisions of draft manuscript: Bigelow G, Ganapathi AM, Brereton RJL

\section{Availability of data and materials}

Not applicable.

\section{Financial support and sponsorship}

None.

\section{Conflicts of interest}

Michael Vallely is a member of the Medtronic North American Surgical Strategic Advisory Board. This did not influence the writing of this manuscript. All other authors declared no conflicts of interest.

\section{Ethical approval and consent to participate}

Not applicable.

\section{Consent for publication}

Written informed consent for the publication of operative images was obtained from patients.

\section{Copyright}

(c) The Author(s) 2021.

\section{REFERENCES}

1. Zhao DF, Edelman JJ, Seco M, et al. Coronary artery bypass grafting with and without manipulation of the ascending aorta: A network meta-analysis. J Am Coll Cardiol 2017;69:924-36. DOI PubMed

2. Neumann FJ, Sousa-Uva M, Ahlsson A, et al; ESC Scientific Document Group. 2018 ESC/EACTS Guidelines on myocardial revascularization. Eur Heart J 2019;40:87-165. DOI PubMed

3. Indja B, Seco M, Seamark R, et al. Neurocognitive and psychiatric issues post cardiac surgery. Heart Lung Circ 2017;26:779-85. DOI PubMed

4. Gaudino M, Antoniades C, Benedetto U, et al; ATLANTIC (Arterial Grafting International Consortium) Alliance. Mechanisms, consequences, and prevention of coronary graft failure. Circulation 2017;136:1749-64. DOI PubMed

5. Taggart DP, D'Amico R, Altman DG. Effect of arterial revascularisation on survival: a systematic review of studies comparing bilateral and single internal mammary arteries. Lancet 2001;358:870-5. DOI PubMed

6. Taggart DP, Benedetto U, Gerry S, et al; Arterial Revascularization Trial Investigators. Bilateral versus single internal-thoracic-artery grafts at 10 years. $N$ Engl J Med 2019;380:437-46. DOI PubMed

7. Taggart DP. Implications of the 10-year outcomes of the Arterial Revascularization Trial (ART) for multiple arterial grafts during coronary artery bypass graft. Eur J Cardiothorac Surg 2019;56:427-8. DOI PubMed

8. Samadashvili Z, Sundt TM, Wechsler A, et al. Multiple versus single arterial coronary bypass graft surgery for multivessel disease. $J$ Am Coll Cardiol 2019;74:1275-85. DOI PubMed

9. Royse AG, Brennan AP, Ou-Young J, Pawanis Z, Canty DJ, Royse CF. 21-year survival of left internal mammary artery-radial arteryY graft. J Am Coll Cardiol 2018;72:1332-40. DOI PubMed

10. Bakaeen FG, Ravichandren K, Blackstone EH, et al. Coronary artery target selection and survival after bilateral internal thoracic artery grafting. J Am Coll Cardiol 2020;75:258-68. DOI PubMed

11. Chikwe J, Lee T, Itagaki S, Adams DH, Egorova NN. Long-term outcomes after off-pump versus on-pump coronary artery bypass grafting by experienced surgeons. J Am Coll Cardiol 2018;72:1478-86. DOI PubMed

12. Benedetto U, Altman DG, Gerry S, et al. Pedicled and skeletonized single and bilateral internal thoracic artery grafts and the incidence of sternal wound complications: Insights from the Arterial Revascularization Trial. J Thorac Cardiovasc Surg 2016;152:270-6. DOI PubMed

13. Vallely MP, Ramponi F, Seco M, Royse A. Multiarterial grafting: Why is it so hard to convince the masses of the benefits? $J$ Thorac Cardiovasc Surg 2020:S0022-5223(20)31553. DOI PubMed

14. Ramponi F, Seco M, Edelman JB, et al. Dual inflow, total-arterial, anaortic, off-pump coronary artery bypass grafting: how to do it. Ann Cardiothorac Surg 2018;7:552-60. DOI PubMed PMC

15. Buxton BF, Hayward PA. The art of arterial revascularization-total arterial revascularization in patients with triple vessel coronary artery disease. Ann Cardiothorac Surg 2013;2:543-51. DOI PubMed PMC

16. Taggart DP. How I deploy arterial grafts. Ann Cardiothorac Surg 2018;7:690-7. DOI PubMed PMC

17. Alsagheir A, Koziarz A, Belley-Côté EP, Whitlock RP. Left atrial appendage occlusion: A narrative review. J Cardiothorac Vasc Anesth 2019;33:1753-65. DOI PubMed 
18. Ross DE. A novel custom-made long shunt simplifies the performance and improves the results of beating-heart surgery. Heart Surg Forum 2003;6:E191-3. PubMed

19. Brereton RJL. Transit time flow measurement in composite arterial revascularisation. Ann Cardiothorac Surg 2018;7:710-5. DOI PubMed PMC

20. Amin S, Pinho-Gomes AC, Taggart DP. Relationship of intraoperative transit time flowmetry findings to angiographic graft patency at follow-up. Ann Thorac Surg 2016;101:1996-2006. DOI PubMed

21. Edelman JJ, Reddel CJ, Kritharides L, et al. Natural history of hypercoagulability in patients undergoing coronary revascularization and effect of preoperative myocardial infarction. J Thorac Cardiovasc Surg 2014;148:536-43. DOI PubMed

22. Gaudino M, Benedetto U, Fremes SE, et al; RADIAL Investigators. Effect of calcium-channel blocker therapy on radial artery grafts after coronary bypass surgery. J Am Coll Cardiol 2019;73:2299-306. DOI PubMed

23. Mack M, Taggart D. Coronary revascularization should be a subspecialty focus in cardiac surgery. J Thorac Cardiovasc Surg 2019;157:945-7. DOI PubMed 